\title{
Development of Communication System Using Eye Movement Extraction via Image Processing with Blink Detection
}

\author{
Masaya Minoda ${ }^{1}$, Takenao Sugi ${ }^{1}$, Yoshitaka Matsuda ${ }^{1}$, Satoru Goto ${ }^{1}$, Takao Yamasaki ${ }^{2}$, \\ Ayame Oishi ${ }^{2}$ and Naruto Egashira ${ }^{3}$ \\ ${ }^{1}$ Department of Advanced Technology Fusion, Saga University, \\ 1 Honjo-machi, Saga 840-8502, Japan \\ ${ }^{2}$ Department of Clinical Neurophysiology, Kyushu University, \\ 3-1-1 Maidashi Higashi-ku, Fukuoka 812-8582, Japan \\ ${ }^{3}$ National Institute of Technology, Kurume College, \\ 1-1-1 Komorino, Kurume, Fukuoka 830-8555, Japan
}

E-mail: sugi@cc.saga-u.ac.jp

\begin{abstract}
In this research, a nurse call system for patients with movement disorders by eye movements extraction based on image processing is developed. In the system of the previous research, to extract the human intention, three kinds of iris positions were detected based on image processing. However, the system has a problem that the detection of eye movement with blink did not work well. In this research, a communication system using eye movement extraction based on image processing with blink detection function is developed. The blink contained in images from camera is detected by using the information about the differences of consecutive images. The accuracy of the proposed method was evaluated through experiments.
\end{abstract}

\section{Introduction}

In general, movement disorders caused by neurodegenerative diseases inhibit the voluntary muscle activity gradually. As the symptom progresses, the communication via utterance, action, equipment operation and so on becomes harder. However, in many cases, the eye movement remains until totally locked-in state even if the symptom progresses. Therefore, the extraction of eye movement is effective as one of the means of communication. From this point of view, a nurse call system for patients with movement disorders by eye movements extraction based on image processing has been developed [1]. In the system, to extract the human intention, three kinds of iris positions (left, right and front) were detected based on image processing. However, this system has a fatal problem that the detection of eye movement with blink did not work well. Therefore, in this research, a communication system using eye movement extraction based on image processing with blink detec- tion function is developed.

\section{Overview of Communication System}

The configuration of the communication system constructed in this research is depicted in Fig. 1.

First, images are acquired from a USB camera, where the images are converted into grayscale ones after importing into a PC. The region of an eye in the image is assigned by dragging mouse on the image.

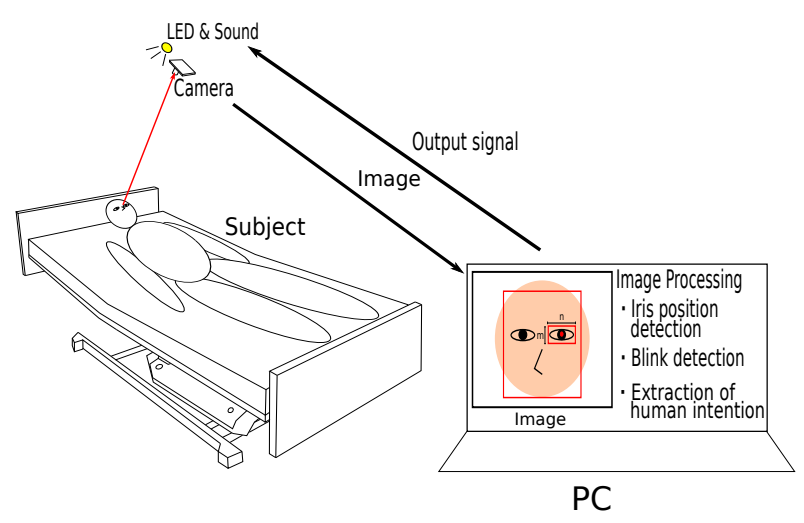

Fig. 1: Communication system

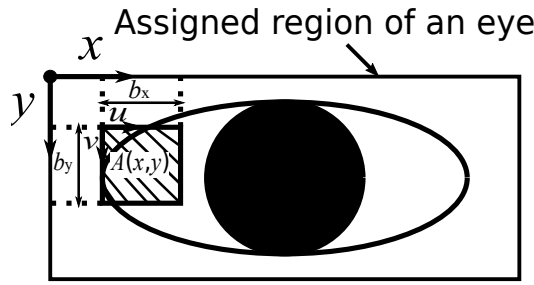

Fig. 2: Detection of iris position 


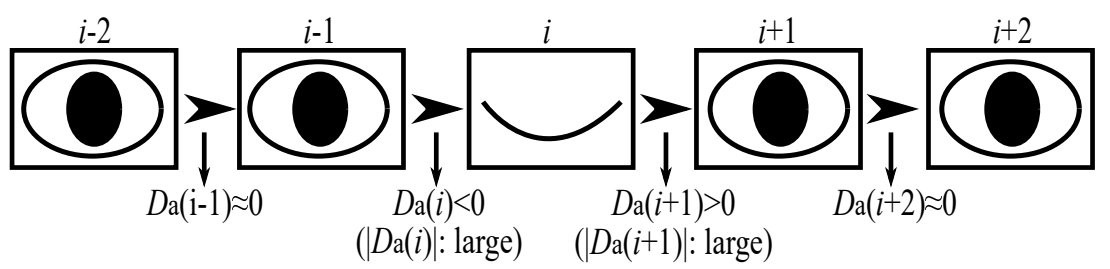

(a) Blink detection for one closed eye image (Pattern 1)

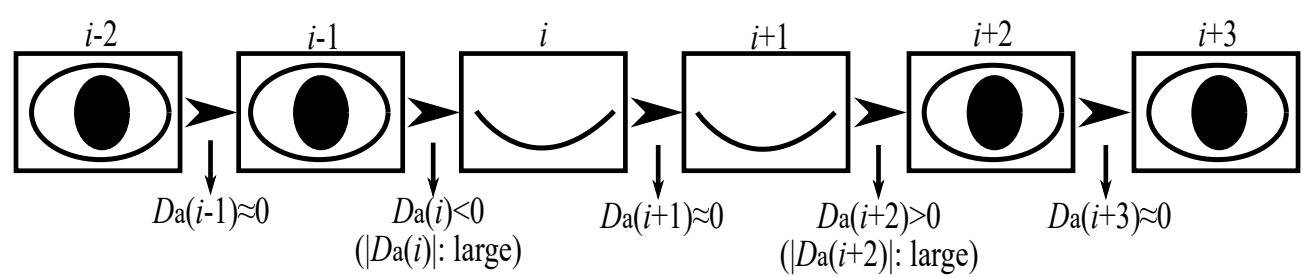

(b) Blink detection for two closed eye images (Pattern 2)

Fig. 3: Blink patterns

For the region $A(x, y)$ with the size $b_{x} \times b_{y}$ at $(x, y)$ as shown in Fig. 2, the sum of intensities $f_{e}(u, v)$ for the pixel at $(u, v)$

$$
T(x, y)=\sum_{(u, v) \in A} f_{e}(u, v)
$$

is calculated. In this research, the position $\left(x_{c}, y_{c}\right)$ of the center of the region $A(x, y)$ such that $T(x, y)$ is minimal is regarded as the iris position [2] since the iris in the assigned region of eye is relatively dark compared with the other parts. Thus, eye movements are traced by calculating the position $\left(x_{c}, y_{c}\right)$ for all images successively. Based on the information of detected eye movements, the user's intention is extracted. Finally, the extracted intention is fed back to the user by LED and/or sound.

It should be pointed out that this system has a serious problem that the user's blinks heavily affect the accuracy of eye movements detection. To solve this problem, in this research, a methodology to remove the influence of blinks on the detection of eye movements is proposed.

\section{Blink Detection}

In this research, the blink detection is realized by the background subtraction method [3] since there is a tendency that blink appears in some successive images as closed or open eye.

The procedure for blink detection proposed in this research is explained below.

First, the difference $D_{a}(i)$ about the intensity $I(x, y, i)$ of the pixel at $(x, y)$ of $i$ th grayscale image:

$$
D_{a}(i)=\sum_{x} \sum_{y}\{I(x, y, i-1)-I(x, y, i)\}
$$

is calculated, where the range of the summation is the assigned region of an eye shown in Fig. 2. In this research, two kinds of blink patterns in Fig. 3 are considered.

Here, let us denote the difference $D_{a}(i)$ satisfying

$$
\left[D_{a}(i+1)-D_{a}(i)\right]\left[D_{a}(i)-D_{a}(i-1)\right]<0
$$

by $P(i)$, where $P(i)$ denotes a peak of (2) [4].

If both

$$
P(i)<-\theta_{b}
$$

and

$$
P(i+1)>\theta_{b}
$$

are satisfied, then it is judged that the images from $(i-1)$ th to $(i+1)$ th contain a blink of Pattern 1 as shown in Fig. 3 (a), where $\theta_{b}$ is the threshold for closed eye detection.

Similarly, if both

$$
P(i)<-\theta_{b}
$$

and

$$
P(i+2)>\theta_{b}
$$

are satisfied, then it is judged that the images from $(i-1)$ th to $(i+2)$ th contain a blink of Pattern 2 as shown in Fig. 3 (b).

Figure 4 is an example of blink detection corresponding to Pattern 1, where (a) is the difference $D_{a}(i),(\mathrm{b})$ is the detection result by the above method and (c) shows images with blink. Figure 4 (a) indicates that both $D_{a}(53)$ and $D_{a}(54)$ satisfy (3) (i.e., $P(53)=D_{a}(53)$ and $\left.P(54)=D_{a}(54)\right)$. Furthermore, both (4) and (5) also hold. Therefore, the judgment of blink detection appeared when the 55th image was processed.

In this research, if a blink is detected, then the iris positions $\left(x_{c}, y_{c}\right)$ obtained from images including the 
Table 1: Experimental condition

\begin{tabular}{|c|c|}
\hline The number of subject & 1 \\
\hline Recording duration & $70[\mathrm{~s}]$ \\
\hline Image size obtained from USB camera & $640 \times 480[\mathrm{pixel}]$ \\
\hline Frame rate & $15[\mathrm{fps}]$ \\
\hline USB camera & DC-NCR300U (Hanwha Q CELLS Japan) \\
\hline Distance between camera and subject & $1.0[\mathrm{~m}]$ \\
\hline Size of $A(x, y): b_{x}, b_{y}$ & $15[\mathrm{pixel}]$ \\
\hline Threshold $\theta_{b}$ & 5000 \\
\hline The number of processed images from video & $350[$ frame $]$ \\
\hline
\end{tabular}

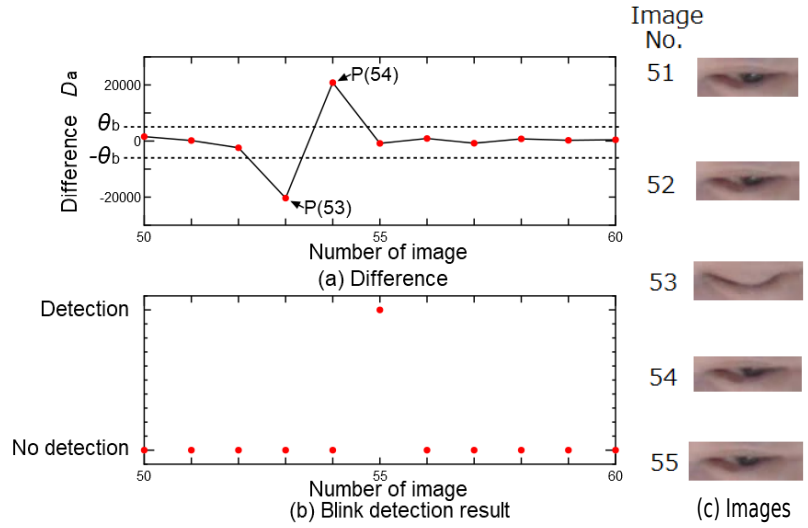

Fig. 4: An example of blink detection

blink are not adopted as the information about the user's intention to be extracted but replaced by the preceding results. For example, in the case of Pattern 1 , the iris position $\left(x_{c}(i), y_{c}(i)\right)$ obtained from the $i$ th image is replaced by $\left(x_{c}(i-1), y_{c}(i-1)\right)$. Similarly, in the case of Pattern 2, the iris positions $\left(x_{c}(i), y_{c}(i)\right)$ and $\left(x_{c}(i+1), y_{c}(i+1)\right)$ obtained from the $i$ th and $(i+1)$ th images are replaced by $\left(x_{c}(i-1), y_{c}(i-1)\right)$.

\section{Experiment}

In order to evaluate the proposed method for the blink detection, an experiment using actual equipment was carried out. The experimental environment is depicted in Fig. 5.

\subsection{Experimental Condition}

The experimental condition is listed in Table 1. The experimental duration was $70[\mathrm{~s}]$, and processed images from USB camera were 350 [frame]. In this experiment, 3 kinds of eye movements (left gaze, right gaze and front gaze) were performed by a healthy subject. Eye movement was started about 30[s] (150[frame] for image).

Then, right gaze and left gaze were repeated alternately, where front gaze was performed between the right gaze and the left gaze. Thus, in the experiment of this research, eye movements for horizontal direction (or $x$-direction) only are dealt with.

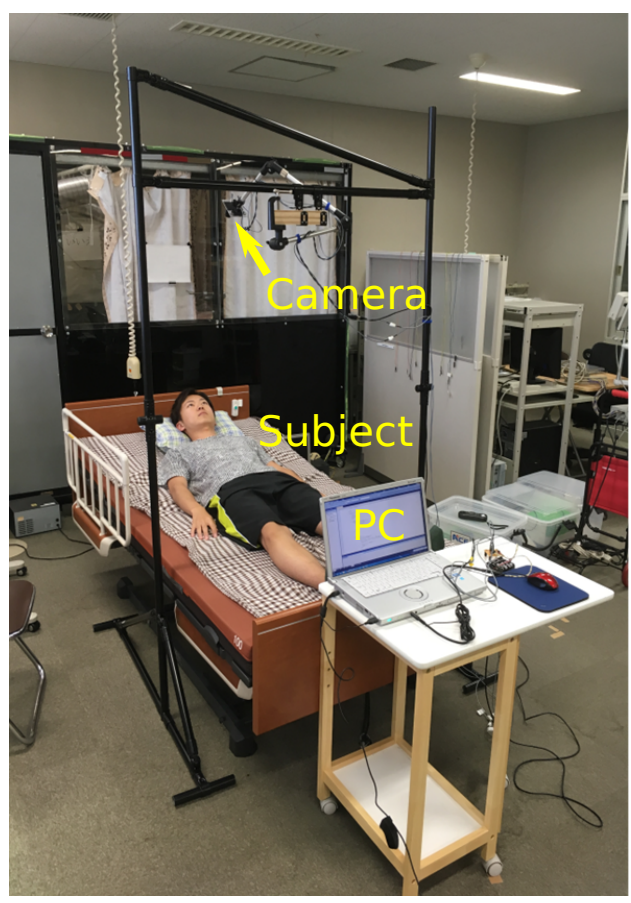

Fig. 5: Experimental environment

\subsection{Experimental Results}

Experimental results are shown in Figs. 6-13. Figure 6 is the calculation results of the difference $D_{a}$. Figure 7 shows the blink detection results by the proposed detection method, where the visual judgment was conducted by visually verifying every image. Figure 8 is the detection results of iris position $x_{c}$ by using the conventional detection method [1] (i.e., without blink detection). On the other hand, Fig. 9 is the detection result of iris position $x_{c}$ by using the method proposed in this research, where, in Figs. 8-9, vertical broken lines are thresholds for the extraction of user's intention. 


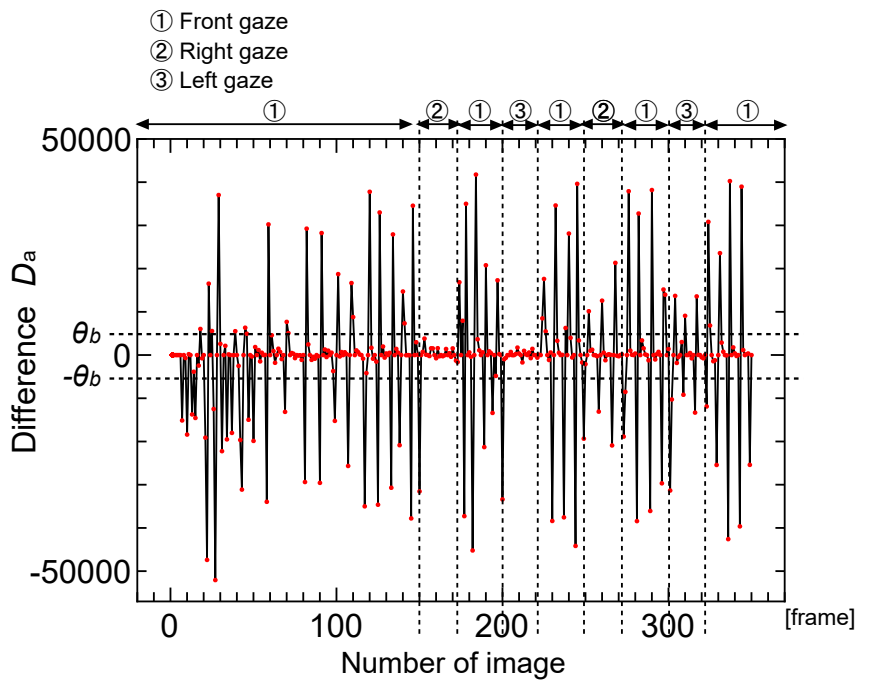

Fig. 6: Difference $D_{a}$ of experiment

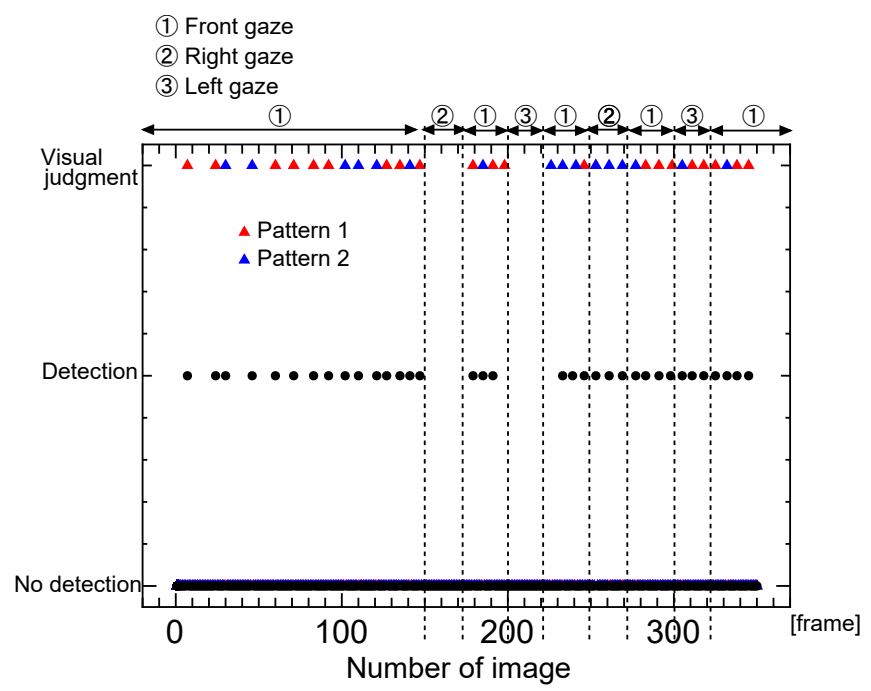

Fig. 7: Blink detection results of experiment

Figures 10-13 are the detailed experimental results from 230 [frame] to 345 [frame] of Figs. 6-9, respectively.

From Fig. 6 (or Fig. 10) we can see that some consecutive peaks for mutually opposite directions appear. This fact justifies the proposed method for blink detection. Furthermore, the frames which appear such peaks almost coincided with those of visual judgment.

As shown in Fig. 7, the number of blinks by visual judgment was 37, and the number of blinks detected by the proposed method was 35 . Therefore, the detection rate of blinks is $35 / 37=94.6[\%]$, and the undetected rate is $2 / 37=5.4[\%]$. Furthermore, there was no over-detected result by the proposed method.

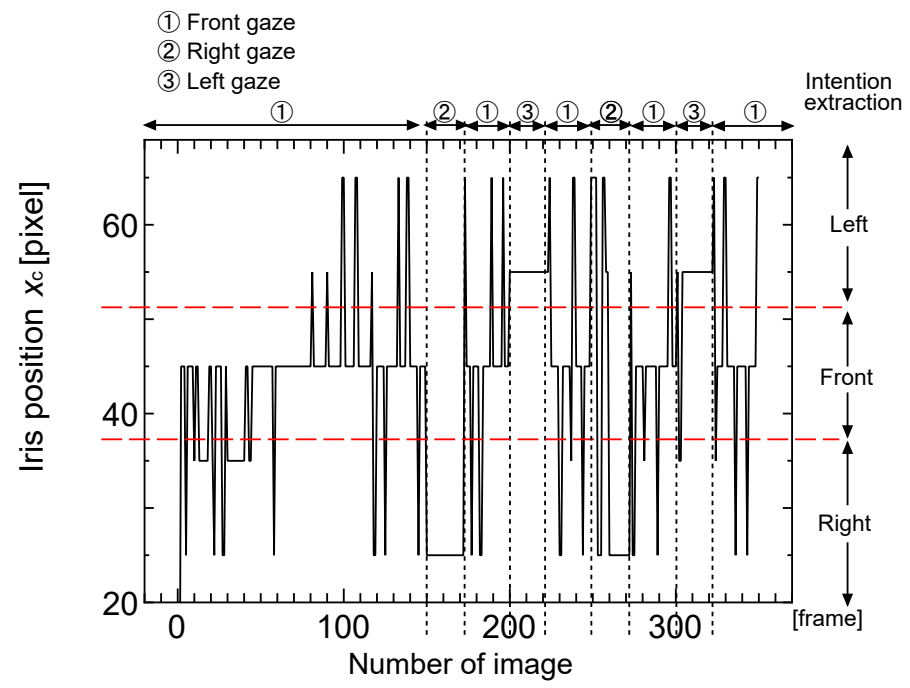

Fig. 8: Iris position $x_{c}$ by conventional method

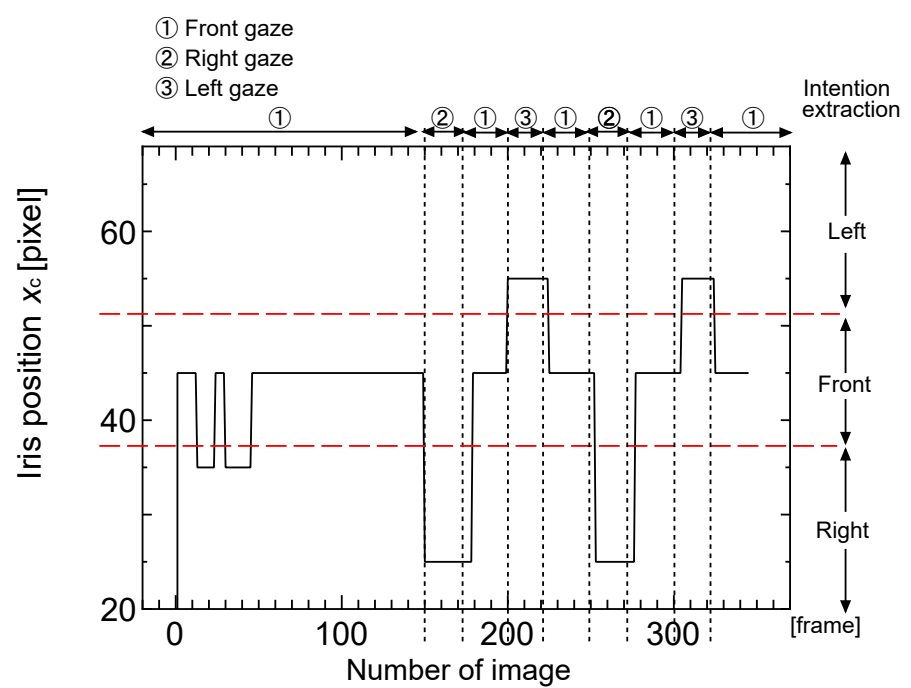

Fig. 9: Iris position $x_{c}$ by proposed method

The undetected reason is that the images captured the half-opened eye and the detection conditions were not satisfied.

The comparison between Fig. 8 (or Fig. 12) and Fig. 7 (or Fig. 11) clarified that the detected iris position $x_{c}$ is heavily influenced by blinks. Therefore, it is difficult to extract the eye movements appropriately by the conventional method.

It is confirmed that the detection results of iris position $x_{c}$ by the proposed method captured eye movements perfectly even if there are some undetected blinks.

Thus, it is concluded from experimental results that 
the proposed system has a sufficient potential as a meaningful communication system using eye movements.

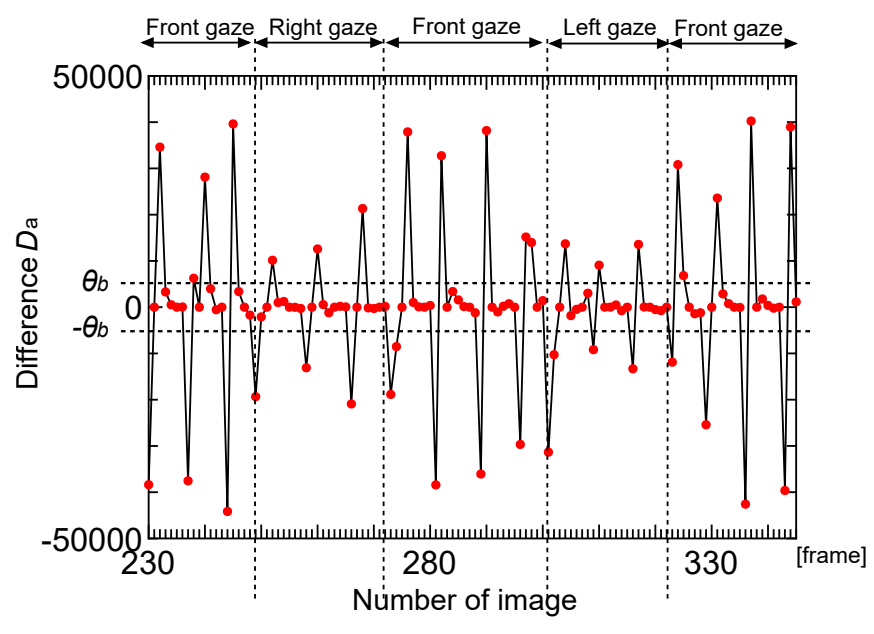

Fig. 10: Difference $D_{a}$ of experiment (detailed)

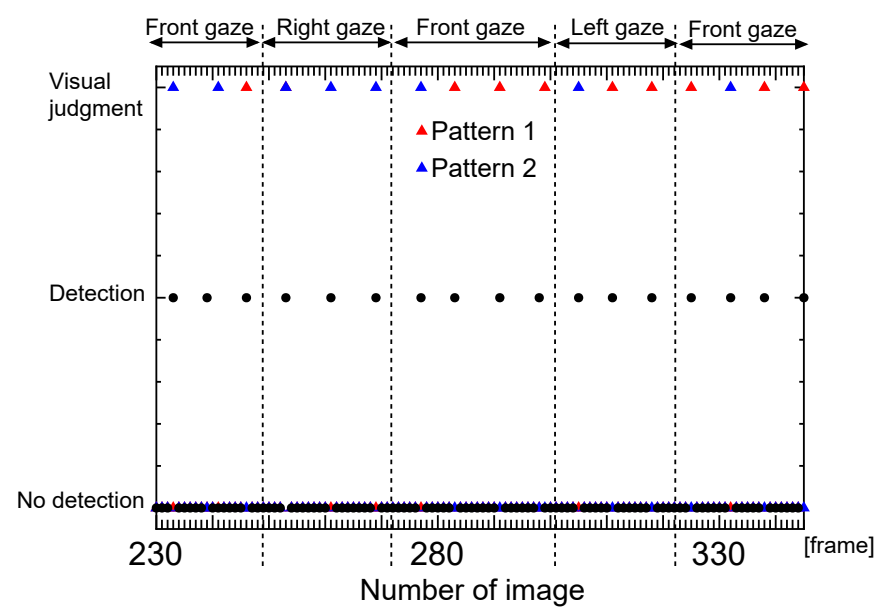

Fig. 11: Blink detection results of experiment (detailed)

\section{Conclusions}

In this research, the development of communication system using eye movements was attempted by image processing with blink detection. The eye movement detection was realized by combining the iris position detection in the previous research with the blink detection via peak extraction based on the background subtraction method. Experimental results demonstrated that the proposed method could bring sufficient accuracy of eye movement extraction by a healthy subject.

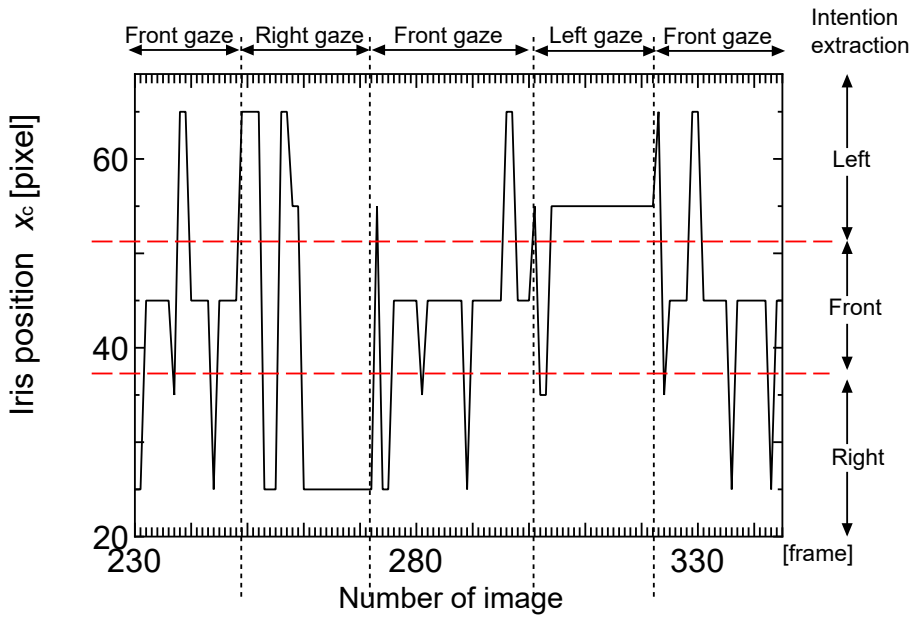

Fig. 12: Iris position $x_{c}$ by conventional method (detailed)

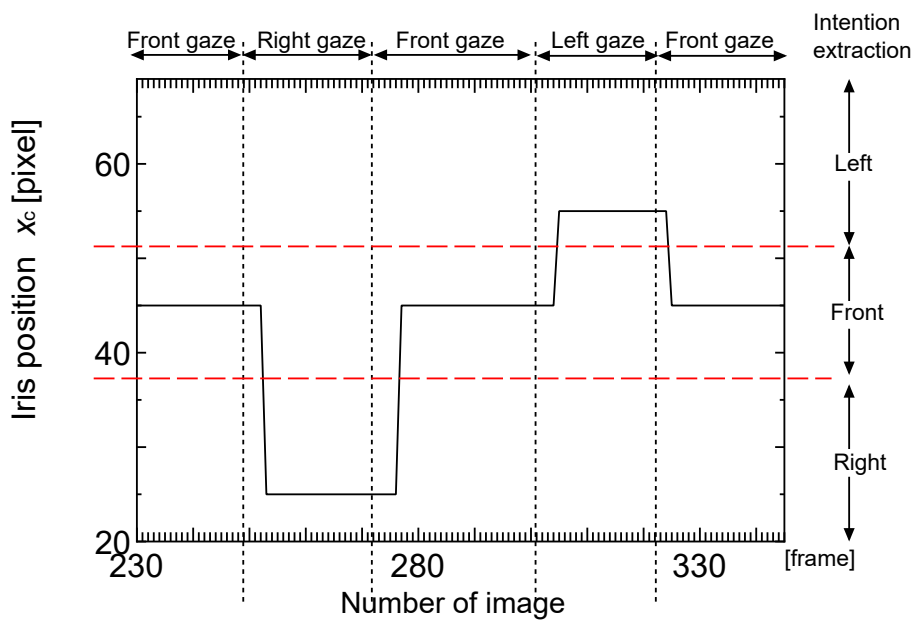

Fig. 13: Iris position $x_{c}$ by proposed method (detailed)

Size, shape and color of human eyes are different for individual subjects. Therefore, to increase a number of subjects are essential for improving the accuracy of the proposed method. In the future research, the proposed system will be applied to patients with movement disorders to verify its effectiveness as a nurse call system.

\section{References}

[1] M. Minoda, T. Sugi, Y. Matsuda, S. Goto, T. Yamasaki and N. Egashira: Communication System by Using Eye Movement Extracted from Image Information, SICE Life Engineering Symposium 
2016, Osaka International House Foundation, Osaka, Japan, 1C1-2, pp. 28-29, 2016 (in Japanese).

[2] E. Watanabe, T. Ozaki and T. Kohama: Analysis of Eye Movements by Interviewer and Interviewee in Interviews, IEICE Technical Report, H2P201443 (2014-9), pp. 1-6, 2014 (in Japanese).

[3] G. Bradski and A. Kaehler: Learning OpenCV, O'REILLY, 2008.

[4] T. Sugi, M. Nakamura, A. Ikeda, T. Nagamine and H. Shibasaki: Real-Time Processing of Blink Artifact Elimination on EEG Records by Use of the Normalized Averaging Method, Japanese Journal of Medical Electronics and Biological Engineering, Vol. 38, No. 1, pp. 33-41, 2000 (in Japanese). 